\title{
Applying fecal microbiota transplantation (FMT) to treat recurrent Clostridium difficile infections (rCDI) in children
}

\author{
Shaaz Fareed ${ }^{1}$, Neha Sarode ${ }^{2}$, Frank J Stewart ${ }^{3}$, Aneeq Malik ${ }^{4}$, Elham Laghaie ${ }^{4}$, Saadia Khizer ${ }^{5}$, Fengxia \\ Yan ${ }^{6}$, Zoe Pratte ${ }^{7}$, Jeffery Lewis ${ }^{8}$, Lilly Cheng Immergluck ${ }^{\text {Corresp. } 1,4}$ \\ 1 Microbiology/Biochemistry/Immunology, Morehouse School of Medicine, Atlanta, GA, United States \\ 2 Department of Organismic \& Evolutionary Biology, Harvard University, Boston, MA, United States \\ 3 Department of Environmental Science \& Technology, Georgia Institute of Technology, Atlanta, GA, United States \\ 4 Clinical Research Center, Morehouse School of Medicine, Atlanta, GA, United States \\ 5 Clinical Research, Children's Healthcare of Atlanta, Atlanta, GA, United States \\ ${ }^{6}$ Community Health \& Preventive Medicine, Morehouse School of Medicine, Atlanta, GA, United States \\ 7 Environmental Science \& Technology, Georgia Institute of Technology, Atlanta, GA, United States \\ 8 Pediatric Gastroenterology, GI Care for Kids, Atlanta, Georgia, United States \\ Corresponding Author: Lilly Cheng Immergluck \\ Email address: limmergluck@msm.edu
}

Background: Fecal Microbiota Transplantation (FMT) is an innovative means of treating recurrent Clostridium difficile infection ( $\mathrm{rCDI}$ ), through restoration of gut floral balance. However, there is a lack of data concerning the efficacy of FMT and its impact on the gut microbiome among pediatric patients. This study analyzes clinical outcomes and microbial community composition among 15 pediatric patients treated for rCDI via FMT.

Methods: This is a prospective, observational, pilot study of 15 children $\leq 18$ years, who presented for rCDI and who met inclusion criteria for FMT at a pediatric hospital and pediatric gastroenterology clinic. Past medical history and demographics were recorded at enrollment and subsequent follow-up.

Specimens of the donor and the patients' pre-FMT and post-FMT fecal specimen were collected and used to assess microbiome composition via $16 \mathrm{~S}$ rRNA gene sequencing.

Results: FMT successfully prevented rCDI episodes for minimum of 3 months post-FMT in all patients, with no major adverse effects. Three patients reported continued GI bleeding; however, all three also had underlying Inflammatory Bowel Disease (IBD). Our analyses confirm a significant difference between preand post-FMT gut microbiome profiles (Shannon diversity index), whereas no significant difference was observed between post-FMT and donor microbiome profiles. At the phyla level, post-FMT profiles showed significantly increased levels of Bacteroidetes and significantly decreased levels of Proteobacteria. Subjects with underlying IBD showed no difference in their pre-and post-FMT profiles.

Conclusion: The low rate of recurrence or re-infection by $C$. difficile, coupled with minimal adverse effects post-FMT, suggests that FMT is a viable therapeutic means to treat pediatric rCDI. Post-FMT microbiomes are different from pre-FMT microbiomes, and similar to those of healthy donors, suggesting successful establishment of a healthier microbiome. 
1 Applying Fecal Microbiota Transplantation (FMT) to Treat Recurrent Clostridium difficile

2

3

4

5 9 GA.

\section{Infections (rCDI) in Children}

${ }^{4}$ Clinical Research Center, Morehouse School of Medicine, Atlanta, GA.

${ }^{5}$ Clinical Research, Children's Healthcare of Atlanta, Atlanta, GA.

${ }^{6}$ Community Health \& Preventive Medicine, Morehouse School of Medicine, Atlanta, GA.

${ }^{7}$ Pediatric Gastroenterology, GI Care for Kids, Atlanta, GA.

*Co first authorship

Corresponding author: Lilly Immergluck, MD, MS, FAAP

Email address: limmergluck@msm.edu 


\section{Introduction}

Childhood infections by the anaerobic gram-positive bacterium Clostridium difficile pose

a significant health challenge, with limited viable treatment options for recurrent infections. Approximately half a million Clostridium difficile infections (CDI) occur annually in the United States and are associated with approximately 29,000 deaths.(Lessa et al. 2015) Although an estimated 17,000 CDI cases involve children ages 1 to 17 years,(Centers for Disease Control and Prevention 2015) the majority of studies exploring treatment efficacy focus on adults (Lees et al. 2016) Even though clinical disease is not seen frequently among infants and very young children, presumably due in part to the lack of toxin-binding receptors in this age group coupled with protective maternal antibodies, the incidence among children has continued to increase over the past two decades (Nylund et al. 2011; Zilberberg et al. 2010). Risks associated with CDI include prior antibiotic use (Schutze et al. 2013) and gut motility dysfunction, with higher incidence observed in children with inflammatory bowel disease (IBD)(Pascarella et al. 2009). Recurrence of disease also has increased, with rates estimated to be as high as 25\% (Aslam et al. 2005). Currently, the preferred first line treatment for recurrent CDI (rCDI) is oral vancomycin (Leong \& Zelenitsky 2013; Sandora TJ 2011). However, up to $25 \%$ of patients undergoing oral vancomycin therapy for rCDI relapse within one month of treatment (Louie et al. 2011; Vincent \& Manges 2015). Furthermore, dysbiosis in gut commensal bacteria, caused initially by $C$. difficile infection, is further exacerbated by antibiotic use (Isaac et al. 2017).

The human gut microbiome is a topic of extensive interest given overwhelming evidence for the microbiome's role in shaping health and disease (Dore \& Blottiere 2015; Estrela et al. 2015; Lozupone et al. 2012). Bacteroidetes and Firmicutes are the major gut phyla of interest: they compose $>90 \%$ of the healthy human gut microbiome, and as commensal bacteria, function as a 
50 barrier in preventing the $C$. difficile overgrowth (Bien et al. 2013; Malys et al. 2015). Studies

51 profiling rCDI gut microbiomes show overall decreased species richness, decreased ratios of

52 Bacteroidetes to Firmicutes, and increased Proteobacteria,(Chang et al. 2008; Theriot \& Young

53 2015) suggesting attempts to correct microbiome dysbiosis as a potential treatment strategy for 54 rCDI.

For children, the repeated bouts of diarrhea, abdominal pain, and medical treatment associated with rCDI incur costs and interfere with normal function. One innovative approach to re-establish the gut microbiome is through fecal microbiota transplantation (FMT). In a review of 317 adult rCDI patients (average age of 53 years) treated via FMT, FMT alleviated rCDI symptoms in $92 \%$ of cases (Gough et al. 2011). Furthermore, FMT has been shown to successfully address dysbiosis by improving the ratio of Bacteroidetes and Firmicutes, thus producing a post-FMT gut bacterial community similar to that of the donor (Seekatz et al. 2014). In general, the pediatric microbiomes not only differ in the proportions of major gut phyla (Hollister et al. 2015), but also exhibit significantly higher interpersonal variation and lower diversity compared to adult microbiomes (Yatsunenko et al. 2012). Though FMT has showed promising results in adults (Rao \& Safdar 2016), this treatment option has been applied sparingly in children (Russell et al. 2010), and even fewer studies have analyzed the efficacy of FMT to treat pediatric rCDI and the impact of the procedure on pediatric gut microbiomes (Hourigan \& Oliva-Hemker 2016a). assess the practicality, safety and efficacy of FMT as a treatment option for pediatric patients with rCDI. A secondary aim was to observe the gut microbiota changes elicited by FMT within pediatric rCDI patients. There have been 45 cases using FMT in children with rCDI and the largest series involved 10 children, 3 of whom had IBD (Kronman et al. 2015). To our knowledge, ours is the 
73 largest FMT study to be conducted among pediatric patients with rCDI, which involves children

74 both with and without underlying inflammatory bowel disease.

75

76

77

\section{$\underline{\text { Materials \& Methods }}$}

Overall Study Design: This is an observational epidemiology study to look at children who failed multiple courses of standard antibiotic therapy for rCDI and elected to undergo FMT. Prior to sampling, this study was approved by the institutional review board of Children's Healthcare of Atlanta and Morehouse School of Medicine.

Study participants ( $\mathrm{n}=15$ pediatric patients) were screened and recruited from a pediatric hospital (Children's Healthcare of Atlanta Hospital at Scottish Rite) and a private pediatric gastroenterology clinic (GI Care for Kids) in Atlanta, Georgia. All study participants were children (age range 21 months to 18 years) who presented with symptoms consistent with rCDI, as determined from examination by the same pediatric gastroenterologist. Patients were eligible for the FMT procedure if they fit the study definition of $\mathrm{rCDI}$ : ongoing diarrhea, a positive $C$. difficile fecal specimen test (polymerase chain reaction assay to detect $C$. difficile toxin genes), and history of failed CDI treatment with antibiotics for a minimum of two prior CDI episodes, of which, at least one episode was treated with a course of oral vancomycin within the previous 8 weeks (Cohen et al. 2010). All FMT procedures were performed via either colonoscopy or nasojejunal tube by the same pediatric gastroenterologist. Previous episodes of CDI were recorded if patient had a documented positive $C$. difficile toxin PCR test and a diagnosis of CDI in his/her medical records. Informed consent (or assent when age appropriate) was obtained from a patient's parent or legal guardian prior to the FMT procedure. A survey questionnaire was administered in a personal interview from study staff to record participant demographic information (age, gender, 
96

97

race/ethnicity), underlying chronic health conditions, prior antibiotic use within 12 months of the time of enrollment, and CDI related symptoms. The following information was also collected from each participant's medical records: nature of prior episodes of CDI, dates of positive PCR $C$. difficile toxin tests, and provider notes documenting history of infections that required antibiotics and specific symptoms consistent with CDI. Five donor samples were ordered from OpenBiome ${ }^{\mathrm{TM}}$ (Medford, MA) to be used for the FMT procedure. All donors were non-family related and underwent laboratory screening by Openbiome ${ }^{\mathrm{TM}}$ (Ling K et al. 2016; Osman et al. 2016) for infectious risk factors (including MDRO's, serologic testing, and fecal specimen testing) and potential microbiome-mediated conditions.

FMT Procedure: FMT procedure was performed on subjects via colonoscopy $(\mathrm{n}=14)$ and nasojejunal tube $(\mathrm{n}=1)$. Prior to FMT, all patients undergoing a colonoscopy completed a standard bowel prep using Polyethylene Glycol 3350. Vancomycin was discontinued at least 48 hours prior to the FMT. Colonoscopies were performed under general anesthesia or under conscious sedation. Up to $240 \mathrm{ml}$ of donor fecal specimen was instilled into the terminal ileum and cecum during colonoscopy in 14 of 15 patients. In one patient, who had undergone colonoscopy due to an underlying disease, $60 \mathrm{cc}$ of donor fecal specimen was instilled via nasojejunal tube into the proximal jejunum.

Follow-up: Follow-up telephone calls were made by research staff after 24 hours and one-week post-FMT procedure. Survey questionnaires were administered by research staff at 1 month and $\geq$ 3 months post-FMT time points, alongside scheduled evaluation by single pediatric gastroenterologist. Responses to questions about study participant's symptoms, including fever, vomiting, abdominal pain and distention, evidence of allergic reaction or bloody fecal specimens were recorded in a relational database. If patients were not available to respond at the 3-month 
119 follow-up visit, attempts to collect a response were performed for up to 15 months after the 120 procedure. (Although fecal samples were collected outside of the 3-month study period, patients

121 were not clinically re-evaluated for evidence of rCDI beyond 3 months of the date of fecal

122 microbiota transplantation.)

123 Fecal Specimen Collection: Three fecal specimen samples were collected for each study 124 participant: 1) from the donor - used for transplant, 2) from the study participant before transplant 125 (pre-FMT), 3) from the study participant following the FMT procedure (at least 3 months after 126 FMT procedure), with the latter used to analyze the effect of FMT on gut microbiome composition. 127 Fecal samples were de-identified and stored in a $-80^{\circ} \mathrm{C}$ freezer until DNA extraction.

128 Fecal Specimen DNA Extraction: DNA was extracted from fecal specimen samples using the MO 129 BIO PowerSoil DNA extraction kit (MOBIO, Carlsbad, CA, USA) following manufacturer 130 instructions, with modifications as recommended by the Human Microbiome Project (HMP) 131 Manual of Procedures (version 12.0). Briefly, before step 2 of the standard protocol, the 132 PowerBead tubes containing samples were heated at $65^{\circ} \mathrm{C}$ for 10 minutes, followed by a second 133 incubation at $95^{\circ} \mathrm{C}$ for 10 minutes to improve cell lysis. The manufacturer protocol was followed 134 for the remainder of the procedure, except for step 12, during which the tubes were centrifuged for 1352 minutes. DNA was extracted from triplicate subsamples for each fecal specimen sample, with 136 the extracted DNA then pooled for quantification and processing. DNA concentrations were 137 measured using the Qubit 2.0 DNA quantification system (ThermoFisher Scientific, MA, USA).

138 16S rRNA Gene PCR and Amplicon Sequencing: Microbiome taxonomic composition in donor, 139 pre-FMT, and post-FMT fecal specimen was characterized by Illumina sequencing of PCR 140 amplicons encompassing the V4 hypervariable region of the 16S rRNA gene. Amplicons were 141 synthesized using Platinum PCR Supermix (ThermoFisher Scientific, MA, USA) with V4-specific 
142 primers F515 and R806(Caporaso et al. 2010), and uniform amounts of DNA template per reaction.

143 Both forward and reverse primers were barcoded and appended with Illumina-specific adapters,

144 as per instructions by Kozich et al (Kozich et al. 2013). Thermal cycler conditions were as follows:

145 initial denaturation at $94^{\circ} \mathrm{C}(3 \mathrm{~min})$, followed by 30 cycles of denaturation at $94^{\circ} \mathrm{C}(45 \mathrm{sec})$, primer

146 annealing at $55^{\circ} \mathrm{C}(45 \mathrm{sec})$ and primer extension at $72^{\circ} \mathrm{C}(90 \mathrm{sec})$, and a final extension at $72^{\circ} \mathrm{C}$

147 for $10 \mathrm{~min}$. Amplicons were verified on agarose gel electrophoresis for size ( $400 \mathrm{bp})$ and purified

148 using Diffinity RapidTip2 pipet tips (Diffinity Genomics, PA, USA). Barcoded and Illumina

149 adaptor-appended amplicons for each sample were pooled at equimolar concentrations and

150 sequenced on an Illumina MiSeq using a 500 cycle v2 reagent kit (250bp paired end) with $15 \%$

151 PhiX genomic library addition to increase read diversity.

152 Statistical and Diversity Analyses: Demultiplexed amplicon read pairs were quality trimmed with

153 Trim Galore! (Babraham Bioinformatics), using a base Phred33 score threshold of Q25 and a

154 minimum length cutoff of $100 \mathrm{bp}$. High quality paired reads were then merged using the software

155 FLASH (Magoč \& Salzberg 2011). Merged reads were analyzed using QIIME v1.9.0 (Caporaso

156 et al. 2010), according to standard protocols. Briefly, reads were screened for chimeras using

157 QIIME's identify_chimeric_seqs.py script with Usearch61 (Edgar 2010). Non-chimeric sequences

158 were clustered into Operational Taxonomic Units (OTUs) at 97\% sequence similarity using

159 UCLUST (Edgar 2010) based on open-reference OTU picking with the script

160 pick_open_reference_otus.py. Taxonomy was assigned to representative OTUs from each cluster

161 using the Greengenes database (Aug 2013 release) (DeSantis et al. 2006).

162 R (R Core Team 2017) packages Phyloseq (McMurdie \& Holmes 2013) and Vegan (Jari Oksanen

163 2017) were used to measure alpha and beta diversity, respectively. Alpha diversity was estimated

164 by the number of observed unique OTUs and the Shannon diversity index at an even sampling 
165 depth of 27,700 sequences. OTUs observed across at least 70\% samples were used for identifying

166 shared OTUs between groups. Microbiome compositional differences among samples were

167 assessed using non-metric multidimensional scaling (NMDS) based on Bray-Curtis distances at

168 OTU level.

169 The significance of the effect of group status (donor, pre-FMT, post-FMT) on microbiome 170 composition was assessed using the envfit function in Vegan, with 999 permutations. Wilcoxon 171 signed rank test with Benjamini-Hochberg correction for multiple hypothesis testing was used to 172 compare the taxonomic differences between pre- and post- FMT, pre- FMT and donor as well as 173 post-FMT and donor. The differences between IBD and non-IBD were compared using Wilcoxon 174 two sample test for demographic information (age, gender, race/ethnicity), underlying chronic 175 health conditions, prior antibiotic use within 12 months of the time of enrollment, and CDI related 176 symptoms and participant's medical records: Nature of prior episodes of CDI, dates of positive 177 PCR, C. difficile toxins, and provider notes documenting history of infections which required 178 antibiotics and specific symptoms consistent with CDI. R and SAS 9.4 were used to perform all 179 the statistical tests and adjusted p-value $<0.05$ (after multiple hypothesis correction) was reported 180 as statistically significant.

\section{Results}

182 Patient Enrollment/Population Characteristics: Fifteen pediatric study participants (8 female, 7 male) were enrolled in this study. Of the potentially 45 specimens, only 40 fecal bio specimens were collected from these 15 patients. (Three post-FMT fecal samples were lost to follow up (LTFU), and two Pre-FMT samples did not yield enough DNA for analysis.) The median age of patients was 8 years (range: 21 months to 18 years). (Table 1) Patients had an average of 3 episodes 
187 of CDI prior to FMT (range: 2 to 5 CDI episodes.). On average, post-FMT fecal samples were 188 collected 6 months after the FMT procedure (range: 3 to 14 months) (Figure 1).

189 Clinical Outcomes: All 15 patients tolerated the FMT procedure without complications 190 immediately post procedure. For the 12 patients who completed their 3-month follow-up 191 appointments, all experienced clinical resolution of CDI and had no recurrent episodes within the 192 duration of the follow-up period (3 months). Abdominal pain was reported by 5 patients (none had 193 underlying IBD) at the 3-month visit; however, the treating pediatric gastroenterologist attributed 194 the pain to other causes, including functional pain and irritable bowel syndrome not related to CDI. 195 All 6 patients with underlying ulcerative colitis $(n=5)$ or Crohn's disease $(n=1)$ reported 196 hematochezia and ongoing abdominal distension beyond 3 months of their FMT date.

197 Microbiome Analysis - Overall Bacterial Community Diversity and Richness: FMT significantly 198 altered gut microbiome composition in all the participants. Alpha diversity (within sample 199 diversity), measured as both observed OTU richness and Shannon diversity, differed between all 200 compared FMT groups, with diversity in the donor sample higher compared to the matched pre201 and post-FMT samples collected from recipients; pre-FMT fecal samples had the lowest diversity. 202 There was no significant difference in observed OTU richness between the five donor samples 203 (Observed OTU p-value = 0.07894; Shannon diversity p-value 0.04306). Post-FMT microbiomes, 204 although more diverse compared to pre-FMT microbiomes, were less diverse than donor 205 microbiomes (Figure 2a). A significant difference (Kruskal-Wallis test, p-value $<0.001$ ) was also 206 observed in shared OTU's between all three groups (donor, recipient pre-FMT, and recipient post207 FMT), where within each comparison, donor vs. post FMT profiles showed the smallest variance 208 (calculated using Wilcoxon signed rank test), while pre vs post FMT profiles had the greatest 209 variance. 

influenced microbiome beta diversity (between sample diversity), as measured by Bray-Curtis distance-based NMDS ordinations ( $\mathrm{r} 2=0.65$, $\mathrm{p}$-value $<0.001$; envfit function in Vegan), with post-FMT microbiome composition being more like that of donor than that of the pre-FMT group

(Figure 2b). Together, these results indicate a positive shift in microbial community composition in response to FMT, suggesting a restructuring to a non-disease state akin to the donor. of interest varied substantially in response to FMT (Figure 3). Notably, the average ratio of Bacteroidetes to Firmicutes (B:F), the predominant phylum-level indicator of a 'normal/healthy' gut microbiome, increased over 7-fold from pre-FMT (average B:F of 0.15) to post-FMT microbiomes (average B:F: 1.12), with the recipient post-FMT B:F approaching that of donor communities (average B:F: 1.49) (Table 2). The shift in this ratio was driven largely by changes in the proportional representation of Bacteroidetes, which comprised an average of only $7 \%$ relative abundance across all pre-FMT samples (excluding study participant 15, who was an outlier), but showed a relative abundance of $41 \%$ and $57 \%$ in recipient post-FMT and donor samples, respectively (Table 2). Much of the observed variation within the Bacteroidetes was due to proportional shifts involving a single genus, Bacteroides. In addition to Bacteroidetes, the phyla Proteobacteria and Verrucomicrobia were significantly enriched in donor and post-FMT compared to pre-FMT fecal samples (Figure 3), whereas these three phyla did not differ in abundance between donor and post-FMT fecal samples, highlighting a shift in the microbiome profile, likely caused by FMT, towards that of the donor. representation of microbial phyla with regards to gut microbiome health has been 
233 questioned(Jandhyala et al. 2015) When examining the changes that occurred instead at the genus

234 level, the most noticeable shifts were reflective of the changes that occurred at the phylum level

235 (Table 2). Specifically, the genus Bacteroides showed a considerable and significant increase in 236 recipients' pre- to post-FMT (from average 6.5 to $39.2 \%$ ). The average relative abundance of the 237 genus Clostridium in the donor was $0.02 \%(\min =0.001 \%, \max =0.08 \%)$, consistent with a non238 disease state. In response to FMT, the average relative abundance of Clostridium in FMT recipients 239 fell roughly $80 \%$, from an average of $1 \%$ pre-FMT $(\min =0 \%$, $\max =4.9 \%)$ to $0.16 \%$ post-FMT $240(\min =0 \%, \max =1.58 \%)$. Three of the FMT recipient participants had Clostridium relative 241 abundance of $0 \%, 4$ had very low but detectable percentages $(\leq 0.01 \%)$, while 6 had levels $\geq 0.5 \%$.

242 Genera identified to be altered significantly in relative abundance per FMT status are listed in 243 supplementary table 1.

244 Effect of Underlying IBD: Of the 15 patients enrolled, 10 had no underlying inflammatory bowel 245 disease (IBD), whereas 5 had been diagnosed with IBD (4 ulcerative colitis and 1 Crohn's disease 246 with one patient having had total colectomy). (Table 3) Community taxonomic profiles clustered 247 according to pre- versus post-FMT status, rather than IBD status (Figure 4), suggesting that underlying IBD had little to no effect on the microbial shift observed because of FMT. There was a marginal effect in the Proteobacteria abundance on separation of community with and without

$\operatorname{IBD}\left(\mathrm{r}^{2}=0.54, \mathrm{p}=0.018\right)$

251 Furthermore, no discernable differences in post-procedural symptoms such as abdominal pain, 252 vomiting, or bloody fecal specimens were observed between patients with or without underlying 253 IBD.

254

255

\section{$\underline{\text { Discussion }}$}


256 Fecal microbiota transplantation (FMT) is a novel approach to treating rCDI by restoration of a

257 healthy gut microbiome (Bakken et al. 2011). FMT has become an acceptable therapeutic 258 consideration for adults, particularly after recurrence of CDI (Surawicz et al. 2013). However, few 259 data are available from pediatric studies beyond case reports or small case series for children 260 (Hourigan \& Oliva-Hemker 2016b). Our findings are consistent with what has been reported in 261 adults and children who have undergone FMT using non-familial, non-autologous donors. 262 Specifically, in our study of 15 children, we show that this procedure is well tolerated by children, 263 irrespective of underlying gastrointestinal disorders (ulcerative colitis and Crohn's) and that the 264 taxonomic shift in the gut microbiome to resemble the donor's microbiome is similar regardless 265 of compositional variation in pre-FMT profiles among patients. Moreover, resolution of CDI 266 symptoms seemed to last from at least 3 months to as long as 14 months. gut community has not 'matured' and the diversity of dominant phyla is shifting. In our study, almost $25 \%$ of participants were less than 2 years of age ( 1 of the 4 patients had ulcerative colitis and received FMT at 21 months of age). However, we did not identify any significant differences in the distribution of dominant phyla across the different ages. There are limited data on how FMT (usually transplantation is with adult donors, who have 'mature' microbial profiles) might affect those as young as 2 years of age (Kahn et al. 2012; Kronman et al. 2015; Russell et al. 2014; Walia et al. 2014; Wang et al. 2015).

Similar to what has been observed in larger adult studies (Seekatz et al. 2014) we also found that for most of our pediatric patients, FMT seemed to induce a post FMT microbiome that

277 was significantly different from the pre-FMT microbiome profile, and more similar to the healthy 278 donor profile. It would be interesting to explore serial fecal biospecimens for up to one year post 
279 FMT and compare the trends of the microbial community composition, particularly as it relates to

280 the Bacteroidetes: Firmicutes ratio for both patients with and without underlying gastrointestinal

281 disorders.

282 Overall, pre-FMT profiles showed high abundance of Proteobacteria, with low amounts 283 of Bacteroidetes. Post-FMT profiles showed an increase in Bacteroidetes, with a large decrease in 284 Proteobacteria. These findings are in agreement with results from past studies of adult patients 285 (Seekatz et al. 2014), De'Argenio and Salvatore, for example, found that a high abundance of Proteobacteria pre-FMT is associated with underlying conditions, such as ulcerative colitis and Crohn's disease (D'Argenio \& Salvatore 2015). Furthermore, Proteobacteria is often selected over Bacteroidetes and Firmicutes when colitis is present (Bradley \& Pollard 2017). It is possible that these factors were responsible for the distributions of Proteobacteria observed in this study. of Bacteroidetes from pre- to post-FMT. Although this was not expected, as all other profiles showed an often, large increase in Bacteroidetes abundance, these two patients also demonstrated significant decreases in the abundances of Proteobacteria, thus still appearing more similar to their respective 'healthy' donor profiles. It is likely that an increase in Bacteroidetes was not observed since the pre-FMT profiles of these patients already displayed higher abundances of Bacteroidetes in comparison to the other subjects. the procedure or preponderance of post procedural adverse effects, nor were there any significant differences observed in pre-FMT profiles or distribution of Bacteroidetes, Firmicutes, or Proteobacteria; the shift in major gut phyla post-FMT was similar across all patients. 
301

302

303

304

305

306

307

308

309

310

311

312

313

314

315

316

317

318

319

320

321 Conclusions: In our limited prospective study of children with rCDI, with and without IBD, FMT

322 is efficacious, with minimal adverse effects, and improves the intestinal microbiome in favor of 323

Limitations/Future Directions: Because this was a prospective observational pilot study, microbiome results and statistical implications are limited by a low sample size $(\mathrm{n}=15$ patients). Not all follow up phone calls were completed within the time frame set in the study design, and 5 post-FMT samples were not obtained. Additional variables that could impact the microbiome, such as diet, exercise, environmental factors, puberty, etc., were not collected, although the list of such potential factors could be in practice rather exhaustive. Fecal samples were ultimately analyzed 3 to 12 months' post-procedure due to inconsistencies in patient compliance. Our covariate analysis is also limited by the fact that study surveys were self-reported. We recognize the risks associated with FMT, and although this study shows promising results, we suggest that FMT should only be used once current guidelines of vancomycin therapy have failed. Future randomized control trials of vancomycin taper versus FMT would be beneficial in determining which option is most effective for treating recurrence. Trials with larger sample sizes need to be performed to establish the effect that FMT has on the microbiome. Furthermore, longitudinal studies of pediatric patients would establish long-term efficacy of this treatment option. Given the small number of pediatric studies, establishing a national FMT database would be useful in analyzing the impact and side effects of FMT. Since this study was an observational study, we did not collect information on participants' diet or environmental factors which may play a role in the gut microbiome. However, in future studies these determinants of health are important to consider in patients who undergo FMT and also in characterizing factors which affect the gut microbiome. higher proportion of Bacteroidetes. 
324

325

326

327

328

329

330

331

332

333

334

335

336

337

338

339

340

341

342

343

344

345

346

347

\section{Acknowledgements:}

We would like to thank: Dr. Sangita Ganesh (Georgia Institute of Technology) for her help with sample processing. Ms. Jonelle McKay, Dr. Robert C. Jerris, Dr. Mark Gonzales (Children’s Healthcare of Atlanta's Clinical Microbiology Laboratory), for ?? . Program managers Mr. Anaam Mohammed (Pediatric Emergency Medicine Associates, LLC) and Ms. Victoria Churchill (Morehouse School of Medicine) for co-ordinating the projects across institutions. And finally our heartfelt gratitude to the nurses and providers at GI Care for Kids, Atlanta.

\section{$\underline{\text { References }}$}

Aslam S, Hamill RJ, and Musher DM. 2005. Treatment of Clostridium difficile-associated disease: old therapies and new strategies. Lancet Infect Dis 5:549-557. 10.1016/S1473-3099(05)70215-2

Bakken JS, Borody T, Brandt LJ, Brill JV, Demarco DC, Franzos MA, Kelly C, Khoruts A, Louie T, Martinelli LP, Moore TA, Russell G, Surawicz C, and Fecal Microbiota Transplantation W. 2011. Treating Clostridium difficile infection with fecal microbiota transplantation. Clin Gastroenterol Hepatol 9:1044-1049. 10.1016/j.cgh.2011.08.014

Bien J, Bozko M, N PM, and Bozko P. 2013. Editorial: Gut Microbiota and Gastrointestinal Diseases: to Treat or Not To Treat. Curr Pharm Des.

Bradley PH, and Pollard KS. 2017. Proteobacteria explain significant functional variability in the human gut microbiome. Microbiome 5:36. 10.1186/s40168-017-0244-z

Caporaso JG, Kuczynski J, Stombaugh J, Bittinger K, Bushman FD, Costello EK, Fierer N, Peña AG, Goodrich JK, Gordon JI, Huttley GA, Kelley ST, Knights D, Koenig JE, Ley RE, Lozupone CA, McDonald D, Muegge BD, Pirrung M, Reeder J, Sevinsky JR, Turnbaugh PJ, Walters WA, Widmann J, Yatsunenko T, Zaneveld J, and Knight R. 2010. QIIME allows analysis of highthroughput community sequencing data. Nature methods 7:335-336. 10.1038/nmeth.f.303 
348 Chang JY, Antonopoulos DA, Kalra A, Tonelli A, Khalife WT, Schmidt TM, and Young VB. 349 2008. Decreased Diversity of the Fecal Microbiome in Recurrent Clostridium difficile350 Associated Diarrhea. The Journal of Infectious Diseases 197:435-438. 10.1086/525047

351 Cohen SH, Gerding DN, Johnson S, Kelly CP, Loo VG, McDonald LC, Pepin J, Wilcox MH, 352 Society for Healthcare Epidemiology of A, and Infectious Diseases Society of A. 2010. Clinical 353 practice guidelines for Clostridium difficile infection in adults: 2010 update by the society for 354 healthcare epidemiology of America (SHEA) and the infectious diseases society of America 355 (IDSA). Infect Control Hosp Epidemiol 31:431-455. 10.1086/651706

356 D'Argenio V, and Salvatore F. 2015. The role of the gut microbiome in the healthy adult status. 357 Clin Chim Acta 451:97-102. 10.1016/j.cca.2015.01.003

358 DeSantis TZ, Hugenholtz P, Larsen N, Rojas M, Brodie EL, Keller K, Huber T, Dalevi D, Hu P, 359 and Andersen GL. 2006. Greengenes, a Chimera-Checked 16S rRNA Gene Database and 360 Workbench Compatible with ARB. Applied and Environmental Microbiology 72:5069-5072. 361 10.1128/AEM.03006-05

362 Dore J, and Blottiere H. 2015. The influence of diet on the gut microbiota and its consequences 363 for health. Curr Opin Biotechnol 32:195-199. 10.1016/j.copbio.2015.01.002

364 Edgar RC. 2010. Search and clustering orders of magnitude faster than BLAST. Bioinformatics 365 26:2460-2461. 10.1093/bioinformatics/btq461

366 Estrela S, Whiteley M, and Brown SP. 2015. The demographic determinants of human microbiome 367 health. Trends Microbiol 23:134-141. 10.1016/j.tim.2014.11.005

368 Gough E, Shaikh H, and Manges AR. 2011. Systematic Review of Intestinal Microbiota 369 Transplantation (Fecal Bacteriotherapy) for Recurrent Clostridium difficile Infection. Clinical 370 Infectious Diseases 53:994-1002. 10.1093/cid/cir632

371 Hollister EB, Riehle K, Luna RA, Weidler EM, Rubio-Gonzales M, Mistretta T-A, Raza S, 372 Doddapaneni HV, Metcalf GA, Muzny DM, Gibbs RA, Petrosino JF, Shulman RJ, and Versalovic 373 J. 2015. Structure and function of the healthy pre-adolescent pediatric gut microbiome. 374 Microbiome 3:36. 10.1186/s40168-015-0101-x

375 Hourigan S, and Oliva-Hemker M. 2016a. Fecal microbiota transplantation in children: a brief 376 review. Pediatr Res. 10.1038/pr.2016.48 
377 Hourigan SK, and Oliva-Hemker M. 2016b. Fecal microbiota transplantation in children: a brief

378 review. Pediatr Res 80:2-6. 10.1038/pr.2016.48

379 Isaac S, Scher JU, Djukovic A, Jimenez N, Littman DR, Abramson SB, Pamer EG, and Ubeda C. 380 2017. Short- and long-term effects of oral vancomycin on the human intestinal microbiota. $J$ 381 Antimicrob Chemother 72:128-136. 10.1093/jac/dkw383

382 Jandhyala SM, Talukdar R, Subramanyam C, Vuyyuru H, Sasikala M, and Nageshwar RD. 2015. 383 Role of normal gut microbiota. World J Gastroenterol 21:8787-8803. 10.3748/wkg/v21.i29.8787

384 Jari Oksanen FGB, Michael Friendly, Roeland Kindt, Pierre Legendre, Dan McGlinn, Peter R. 385 Minchin, R. B. O'Hara, Gavin L. Simpson, Peter Solymos, M. Henry, H. Stevens, Eduard Szoecs 386 and Helene Wagner. 2017. vegan: Community Ecology Package.

387 Kahn SA, Gorawara-Bhat R, and Rubin DT. 2012. Fecal bacteriotherapy for ulcerative colitis: 388 patients are ready, are we? Inflamm Bowel Dis 18:676-684. 10.1002/ibd.21775

389 Kozich JJ, Westcott SL, Baxter NT, Highlander SK, and Schloss PD. 2013. Development of a 390 dual-index sequencing strategy and curation pipeline for analyzing amplicon sequence data on the 391 MiSeq Illumina sequencing platform. Appl Environ Microbiol 79:5112-5120. 392 10.1128/AEM.01043-13

393 Kronman MP, Nielson HJ, Adler AL, Giefer MJ, Wahbeh G, Singh N, Zerr DM, and Suskind DL. 394 2015. Fecal microbiota transplantation via nasogastric tube for recurrent clostridium difficile 395 infection in pediatric patients. $J$ Pediatr Gastroenterol Nutr 60:23-26. 396 10.1097/MPG.0000000000000545

397 Lees EA, Miyajima F, Pirmohamed M, and Carrol ED. 2016. The role of Clostridium difficile in 398 the paediatric and neonatal gut - a narrative review. European Journal of Clinical Microbiology 399 \& Infectious Diseases 35:1047-1057. 10.1007/s10096-016-2639-3

400 Leong C, and Zelenitsky S. 2013. Treatment Strategies for Recurrent Clostridium difficile 401 Infection. The Canadian Journal of Hospital Pharmacy 66:361-368.

402 Lessa FC, Mu Y, Bamberg WM, Beldavs ZG, Dumyati GK, Dunn JR, Farley MM, Holzbauer SM, 403 Meek JI, Phipps EC, Wilson LE, Winston LG, Cohen JA, Limbago BM, Fridkin SK, Gerding DN, 404 and McDonald LC. 2015. Burden of Clostridium difficile Infection in the United States. New 405 England Journal of Medicine 372:825-834. 10.1056/NEJMoa1408913 
406 Ling K, Koelsch E, Dubois N, O’Brien K, Stoltzner Z, and P P. 2016. Prospective Laboratory 407 Evaluation of Fecal Microbiota Transplantation Donors: Results From an International Public 408 Stool Bank. Open Forum Infect Dis:2123.

409 Louie TJ, Miller MA, Mullane KM, Weiss K, Lentnek A, Golan Y, Gorbach S, Sears P, and Shue 410 Y-K. 2011. Fidaxomicin versus Vancomycin for Clostridium difficile Infection. New England 411 Journal of Medicine 364:422-431. 10.1056/NEJMoa0910812

412 Lozupone CA, Stombaugh JI, Gordon JI, Jansson JK, and Knight R. 2012. Diversity, stability and 413 resilience of the human gut microbiota. Nature 489:220-230. 10.1038/nature11550

414 Magoč T, and Salzberg SL. 2011. FLASH: fast length adjustment of short reads to improve 415 genome assemblies. Bioinformatics 27:2957-2963. 10.1093/bioinformatics/btr507

416 Malys MK, Campbell L, and Malys N. 2015. Symbiotic and antibiotic interactions between gut 417 commensal microbiota and host immune system. Medicina 51:69-75.

418 McMurdie PJ, and Holmes S. 2013. phyloseq: an R package for reproducible interactive analysis 419 and graphics of microbiome census data. PLoS One 8:e61217. 10.1371/journal.pone.0061217

420 Nylund CM, Goudie A, Garza JM, Fairbrother G, and Cohen MB. 2011. Clostridium difficile 421 infection in hospitalized children in the United States. Arch Pediatr Adolesc Med 165:451-457. 422 10.1001/archpediatrics.2010.282

423 Osman M, O'Brien K, Stoltzner Z, Ling K, Koelsch E, Dubois N, Khoiri A, Amaratunga K, Smith 424 M, and Kassam Z. 2016. Safety and Efficacy of Fecal Microbiota Transplantation for Recurrent 425 Clostridium difficile Infection From An International Public Stool Bank: Results From a 2050426 Patient Multicenter Cohort. Open Forum Infectious Diseases 3:2120-2120. 427 10.1093/ofid/ofw172.1668

428 Pascarella F, Martinelli M, Miele E, Del Pezzo M, Roscetto E, and Staiano A. 2009. Impact of 429 Clostridium difficile infection on pediatric inflammatory bowel disease. J Pediatr 154:854-858. $430 \quad 10.1016 /$ j.jpeds.2008.12.039

431 Center for Disease Control and Prevention. 2015. Nearly half a million Americans suffered from 432 Clostridium difficile infections in a single year. CDC Newsroom. 433 https://www.cdc.gov/media/releases/2015/p0225-clostridium-difficile.html 
434 R Core Team. 2017. R: A language and environment for statistical computing. . R Foundation for 435 Statistical Computing, Vienna, Austria. https://www.R-project.org/

436 Rao K, and Safdar N. 2016. Fecal Microbiota Transplantation for the Treatment of Clostridium 437 difficile Infection. Journal of hospital medicine 11:56-61. 10.1002/jhm.2449

438 Russell G, Kaplan J, Ferraro M, and Michelow IC. 2010. Fecal bacteriotherapy for relapsing 439 Clostridium difficile infection in a child: a proposed treatment protocol. Pediatrics 126:e239-242. $440 \quad 10.1542 /$ peds.2009-3363

441 Russell GH, Kaplan JL, Youngster I, Baril-Dore M, Schindelar L, Hohmann E, and Winter HS. 442 2014. Fecal transplant for recurrent Clostridium difficile infection in children with and without 443 inflammatory bowel disease. J Pediatr Gastroenterol Nutr 58:588-592.

444 Sandora TJ FM, Flaherty K, Helsing L, Scanlon P, Potter-Bynoe G, Gidengil CA, Lee GM. 2011. 445 Epidemiology and risk factors for Clostridium difficile infection in children. Pedatri Infect Dis $J$ 446 7. 10.1097/INF.0b013e31820bfb29.

447 Schutze GE, Willoughby RE, Brady MT, Byington CL, Davies HD, Edwards KM, Glode MP, 448 Jackson MA, Keyserling HL, Maldonado YA, Murray DL, Orenstein WA, and Zaoutis TE. 2013. 449 Clostridium difficile Infection in Infants and Children. Pediatrics 131:196-200. $450 \quad 10.1542 /$ peds.2012-2992

451 Seekatz AM, Aas J, Gessert CE, Rubin TA, Saman DM, Bakken JS, and Young VB. 2014. 452 Recovery of the Gut Microbiome following Fecal Microbiota Transplantation. mBio 5:e0893453 00814. 10.1128/mBio.00893-14

454 Surawicz CM, Brandt LJ, Binion DG, Ananthakrishnan AN, Curry SR, Gilligan PH, McFarland 455 LV, Mellow M, and Zuckerbraun BS. 2013. Guidelines for diagnosis, treatment, and prevention 456 of Clostridium difficile infections. Am J Gastroenterol 108:478-498; quiz 499. 10.1038/ajg.2013.4

457 Theriot CM, and Young VB. 2015. Interactions Between the Gastrointestinal Microbiome and 458 Clostridium difficile. Annual review of microbiology 69:445-461. 10.1146/annurev-micro$459 \quad 091014-104115$

460 Vincent C, and Manges AR. 2015. Antimicrobial Use, Human Gut Microbiota and Clostridium 461 difficile Colonization and Infection. Antibiotics 4:230-253. 10.3390/antibiotics4030230 
462 Walia R, Garg S, Song Y, Girotra M, Cuffari C, Fricke WF, and Dutta SK. 2014. Efficacy of fecal

463 microbiota transplantation in 2 children with recurrent Clostridium difficile infection and its 464 impact on their growth and gut microbiome. J Pediatr Gastroenterol Nutr 59:565-570. 465 10.1097/MPG.0000000000000495

466 Wang J, Xiao Y, Lin K, Song F, Ge T, and Zhang T. 2015. Pediatric severe pseudomembranous 467 enteritis treated with fecal microbiota transplantation in a 13-month-old infant. Biomed Rep 3:173468 175. 10.3892/br.2014.403

469 Yatsunenko T, Rey FE, Manary MJ, Trehan I, Dominguez-Bello MG, Contreras M, Magris M, 470 Hidalgo G, Baldassano RN, Anokhin AP, Heath AC, Warner B, Reeder J, Kuczynski J, Caporaso 471 JG, Lozupone CA, Lauber C, Clemente JC, Knights D, Knight R, and Gordon JI. 2012. Human 472 gut microbiome viewed across age and geography. Nature 486:222-227.

473 Zilberberg MD, Tillotson GS, and McDonald C. 2010. Clostridium difficile infections among 474 hospitalized children, United States, 1997-2006. Emerg Infect Dis 16:604-609. $47510.3201 /$ eid1604.090680 


\section{Table $\mathbf{1}$ (on next page)}

Patient characteristics.

Summary of patient profiles for all subjects enrolled in this study. Included are demographic information, delivery route of fecal microbiota transplantation (FMT), underlying inflammatory bowel disease (IBD), and recorded number of prior Clostridium difficile infections (CDI) episodes. 


\begin{tabular}{|c|c|c|c|c|c|c|c|c|c|}
\hline ID & Sex & Race & $\begin{array}{c}\text { FMT } \\
\text { Delivery } \\
\text { Method }\end{array}$ & $\begin{array}{c}\text { Age } \\
\text { (years) } \\
\text { at } \\
\text { FMT }\end{array}$ & $\begin{array}{c}\text { Time } \\
\text { FMT and } \\
\text { post-FMT } \\
\text { Stool } \\
\text { Collection } \\
\text { (Months) } \\
\end{array}$ & $\begin{array}{c}\begin{array}{c}\text { Number } \\
\text { of }\end{array} \\
\text { Antibiotic } \\
\text { Courses } \\
\text { Prior to } \\
\text { FMT }^{\wedge} \\
\end{array}$ & $\begin{array}{l}\text { Underlying } \\
\text { IBD }\end{array}$ & $\begin{array}{c}\text { Number of } \\
\text { CDI } \\
\text { Episodes } \\
\text { Prior to } \\
\text { FMT }\end{array}$ & $\begin{array}{c}\text { FMT } \\
\text { Donor } \\
\text { ID }\end{array}$ \\
\hline 1 & $\mathrm{~F}$ & Biracial & Colonoscopy & 2 & 3 & 4 & No & 4 & 05 \\
\hline 2 & $\mathrm{~F}$ & Black & Colonoscopy & 7 & 9 & 1 & $\begin{array}{c}\text { Ulcerative } \\
\text { Colitis }\end{array}$ & 3 & 05 \\
\hline 3 & $\mathrm{M}$ & White & Colonoscopy & 16 & 4 & 4 & $\begin{array}{c}\text { Ulcerative } \\
\text { Colitis }\end{array}$ & 4 & 05 \\
\hline 4 & $\mathrm{~F}$ & Biracial & Colonoscopy & 8 & 14 & 3 & No & 3 & 05 \\
\hline 5 & $\mathrm{M}$ & Black & Colonoscopy & 10 & 7 & 4 & $\begin{array}{c}\text { Ulcerative } \\
\text { Colitis }\end{array}$ & 3 & 05 \\
\hline 6 & $\mathrm{~F}$ & Black & $\begin{array}{c}\text { Nasojejunal } \\
\text { Tube }\end{array}$ & 8 & LTFU* & 8 & $\begin{array}{c}\text { Ulcerative } \\
\text { Colitis }\end{array}$ & 3 & 05 \\
\hline 7 & $\mathrm{M}$ & White & Colonoscopy & 1 & 6 & 4 & No & 4 & 37 \\
\hline 8 & $\mathrm{~F}$ & White & Colonoscopy & 7 & 4 & 2 & $\begin{array}{l}\text { Crohn's } \\
\text { Disease }\end{array}$ & 3 & 37 \\
\hline 9 & $\mathrm{~F}$ & White & Colonoscopy & 18 & 4 & 2 & No & 2 & 37 \\
\hline 10 & $\mathrm{~F}$ & White & Colonoscopy & 2 & 6 & 2 & No & 3 & 37 \\
\hline 11 & $\mathrm{~F}$ & White & Colonoscopy & 8 & 6 & 2 & No & 5 & 37 \\
\hline 12 & M & White & Colonoscopy & 2 & LTFU & 2 & No & 2 & 12 \\
\hline 13 & M & Biracial** $^{*}$ & Colonoscopy & 5 & 3 & 3 & No & 3 & 77 \\
\hline 14 & $\mathrm{~F}$ & White & Colonoscopy & 15 & LTFU & 7 & No & 3 & 66 \\
\hline 15 & M & White & Colonoscopy & 10 & 2 & 1 & No & 3 & 77 \\
\hline
\end{tabular}

*LTFU=Sample Lost to Follow Up

**Biracial $=$ Participant self-identified as Black/White

${ }^{\wedge}$ Number of antibiotic courses only within the period of 12 months prior to FMT was included

All patients had up to date vaccinations for routine immunizations, including Diphtheria-Tetanus-acellular Pertussis, Haemophilus Influenza Type B, Influenza, Pneumococcal Conjugate Vaccine, with 7 or 13 Serogroups, Inactivated Polio Virus, Hepatitis A and B;

Rotavirus; and Varicella. 


\section{Table 2 (on next page)}

Microbiome analyses- taxon level changes between Bacteroidetes, Firmicutes, and Proteobacteria

Abundance of major microbial phyla in stool microbiomes, expressed for each sample type as the percentage of total sequences classifiable to the phylum level, averaged across all samples. 


\begin{tabular}{cccc}
\hline Phylum & Donor & Pre-FMT & Post-FMT \\
\hline Bacteroidetes & $57 \%$ & $7 \%$ & $41 \%$ \\
Firmicutes (F) & $38 \%$ & $50 \%$ & $36 \%$ \\
Proteobacteria (B) & $4 \%$ & $41 \%$ & $5 \%$ \\
\hline Average B:F Ratio & 1.49 & 0.15 & 1.12 \\
\hline
\end{tabular}




\section{Table 3 (on next page)}

Comparison of symptoms post fecal microbiota transplantation (FMT) for patients with and without underlying inflammatory bowel diseases (IBD).

Gastrointestinal (GI) symptoms include one or more of the following: abdominal pain, fever, abdominal distension, or bloody stool. (The categorical variables were compared using Fisher's Exact test due to the small sample and the continuous variables were evaluated using Wilcoxon two independent sample test.) 


\begin{tabular}{|c|c|c|c|}
\hline Variable & $\begin{array}{c}\text { No Underlying IBD } \\
(\mathrm{n}=10,66 \%)\end{array}$ & $\begin{array}{c}\text { Underlying IBD } \\
(\mathrm{n}=5, \mathbf{3 3} \%)\end{array}$ & p-value \\
\hline Age, median(IQR) & $6.55(8.01)$ & $8.02(2.61)$ & 0.4053 \\
\hline Gender male\% & $5(50)$ & $2(40)$ & 1.0000 \\
\hline GI symptoms prior to FMT (yes, \%) & $9(90)$ & $5(100)$ & 1.0000 \\
\hline GI symptoms at 24-hour post FMT & & & 0.5055 \\
\hline No & $3(30)$ & $0(0)$ & \\
\hline Yes & $7(70)$ & $4(100)$ & \\
\hline GI symptoms at 1 -week post FMT & & & 0.0667 \\
\hline No & $1(12.5)$ & $3(75)$ & \\
\hline Yes & $7(87.5)$ & $1(25)$ & \\
\hline GI symptoms at 1-month post FMT & & & 0.2657 \\
\hline No & $3(37.5)$ & $4(80)$ & \\
\hline Yes & $5(62.5)$ & $1(20)$ & \\
\hline GI symptoms at 3 months post FMT & & & 1.0000 \\
\hline No & $2(28.6)$ & $1(20)$ & \\
\hline Yes & $5(71.4)$ & $4(80)$ & \\
\hline Route of FMT & & & 0.3333 \\
\hline Colonoscopy & $10(100)$ & $4(80)$ & \\
\hline NJ Tube & 0 & $1(20)$ & \\
\hline $\begin{array}{l}\text { Number of Antibiotic courses prior to FMT, } \\
\text { median(IQR) }\end{array}$ & $2.5(2)$ & $4(2)$ & 0.6217 \\
\hline $\begin{array}{l}\text { Number of ED visits within a year of FMT, } \\
\text { median(IQR) }\end{array}$ & $1.5(3)$ & $4(3)$ & 0.0468 \\
\hline Number of CDI episodes, median(IQR) & $3(1)$ & $3(0)$ & 0.9456 \\
\hline B:F Ratio, median(IQR) & $0.0021(0.0068)$ & $0.0030(0.0027)$ & 0.8209 \\
\hline $\begin{array}{l}\text { Clostridia Distribution, } \\
\text { median(IQR) }\end{array}$ & $0.0055(0.0127)$ & $0.00001(0.0109)$ & 0.4102 \\
\hline
\end{tabular}




\section{Figure 1 (on next page)}

Time of stool collection post-fecal microbiota transplantation (FMT).

Displayed is a histogram detailing how many patients submitted stool at the displayed time periods (in months) Post-FMT. 


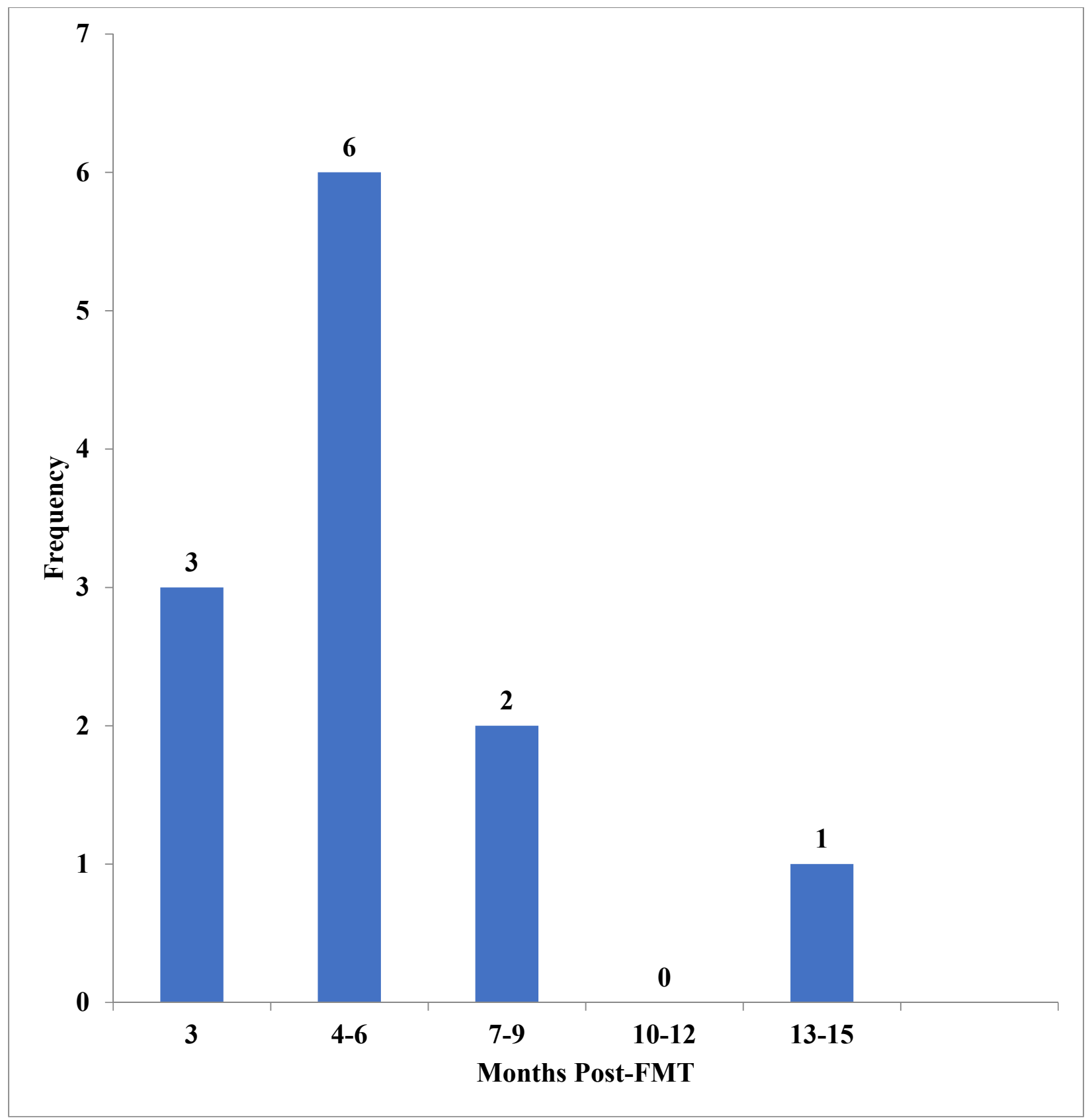




\section{Figure 2}

Alpha and beta diversity of samples

(A) Alpha diversity is a measure of species richness within a sample which is quantitatively expressed here as a box-plot of the number of observed unique taxa/OTU's and Shannon diversity index on y-axis. Individual samples are colored according to their patient ID grouped by their FMT status on the $\mathrm{x}$-axis (B) Beta diversity is a measure of taxonomic composition diversity between sample that is represented as Non-metric multidimensional scaling (NMDS) ordination of samples based on Bray-Curtis distance matrix. The color and shape of samples are according to their FMT status identity $(\mathrm{RED}=$ donor, $\mathrm{BLUE}=$ pre-FMT or GREEN= postFMT), with samples belonging to the same FMT group connected to form polygons. The more similar the groups are to each other (donor and post-FMT), the closer their polygon clusters are going to be on the ordination plot and vice versa (pre-FMT compared to both donor and post-FMT). The dotted lines connect individual samples to the group centroid while the ellipse gives an estimate of standard deviation of the scores. (FMT= Fecal Microbiota Transplantation).
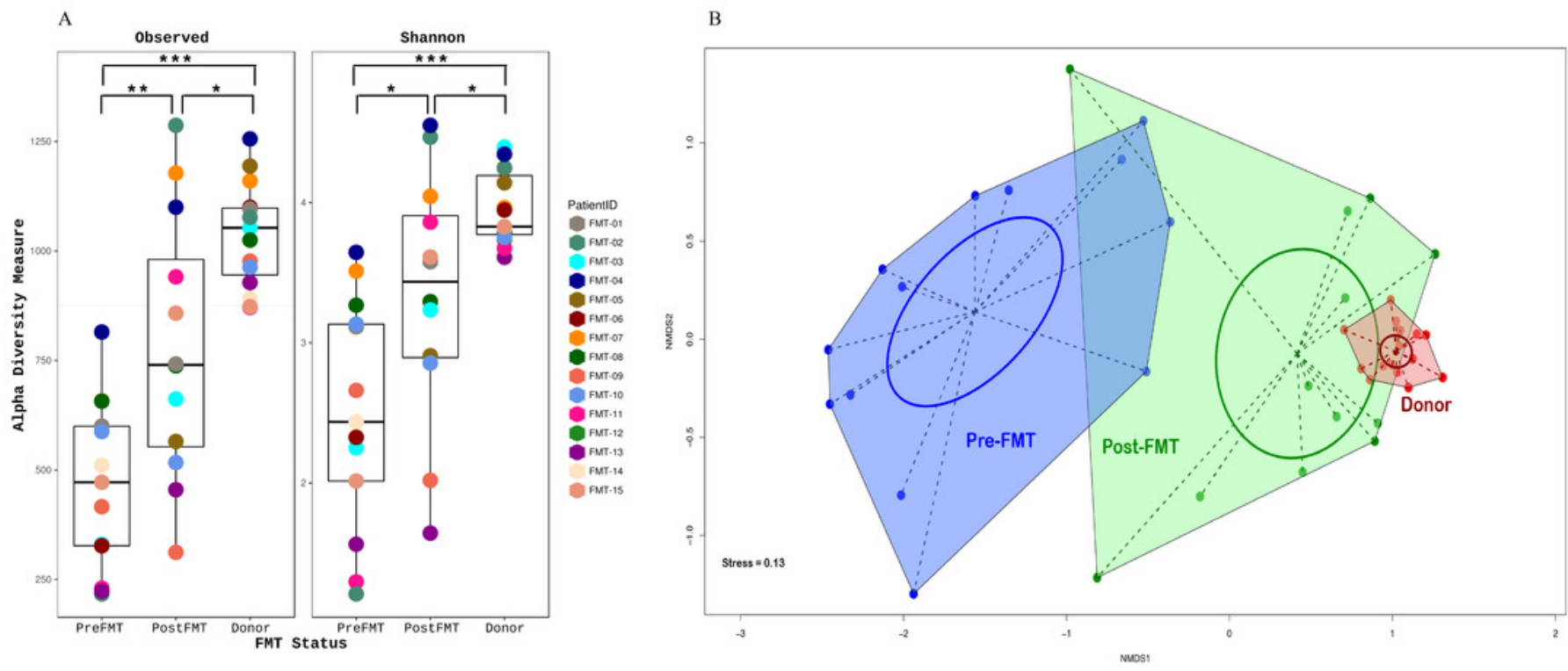


\section{Figure 3}

Distribution of Major Phyla between Donor, Recipient Pre- and Post-FMT Microbiomes.

The bubble plot above displays the proportions of phyla observed in each sample. Red indicates donor samples, blue indicates pre-FMT samples, and green indicates post-FMT samples. An increase in Bacteroidetes can be observed from pre to post-FMT samples, as well as a decrease in Proteobacteria. Post-FMT phyla proportions are more similar to donor profiles. (FMT= Fecal Microbiota Transplantation)

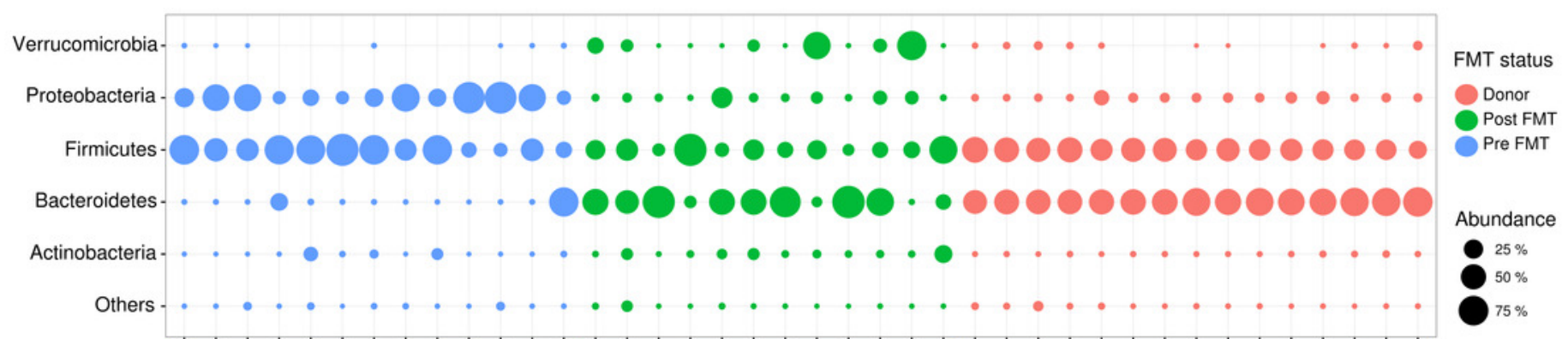




\section{Figure 4}

Effect of inflammatory bowel disease (IBD) on community diversity

NMDS ordination of samples considering their IBD status showed that, underlying IBD condition had no significant effect on community dynamics. Samples are colored based on the combination of their FMT and IBD status, with samples belonging to the same group connected with polygon for visual clarity. (FMT=Fecal Microbiota Transplantation). 
Recipient no IBD, pre-FMT

Recipient with IBD, pre-FMT
Recipient no IBD, post-FMT Recipient with IBD, post-FMT

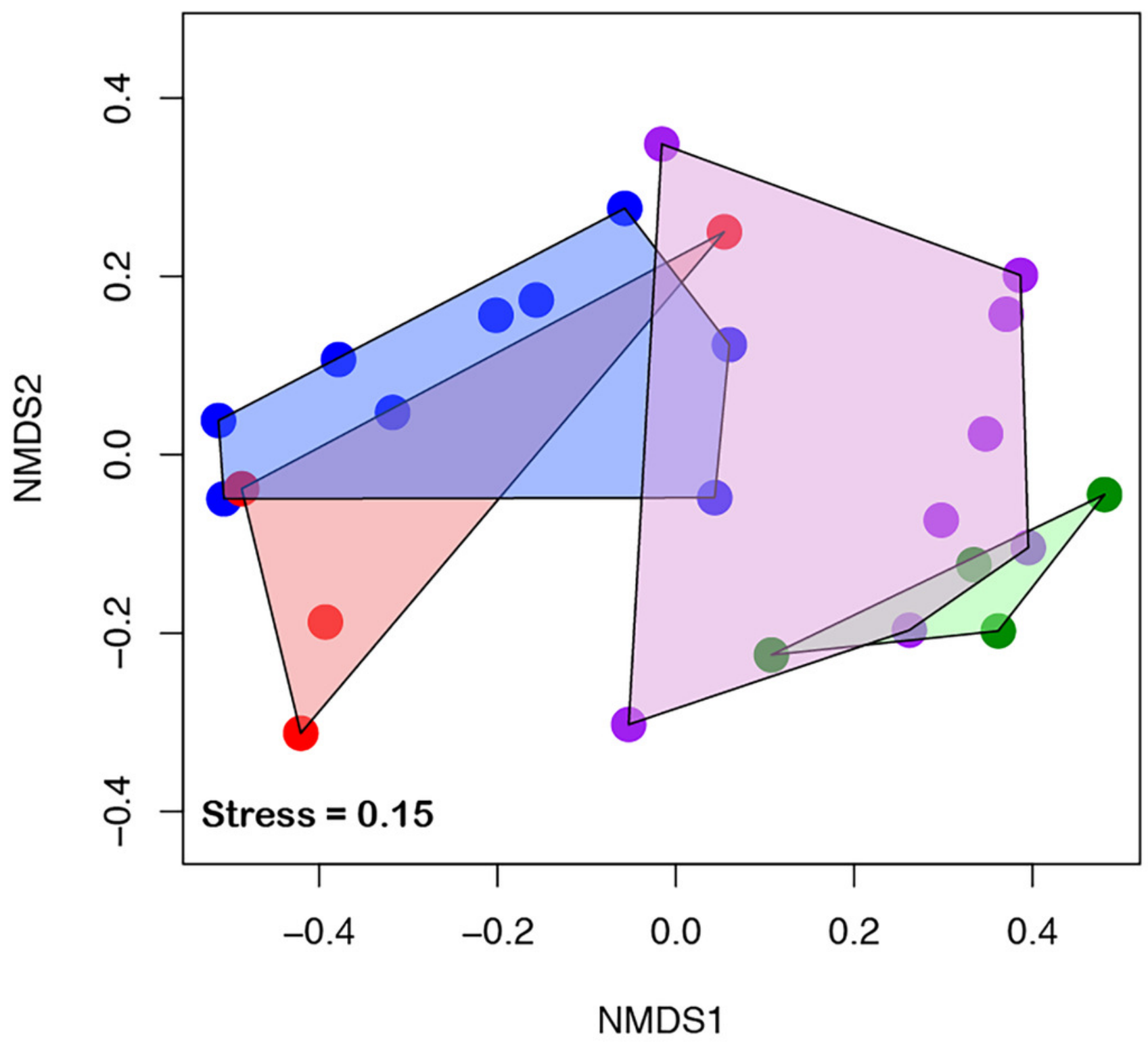

